\title{
Necrotizing fasciitis and necrotizing pneumonia caused by Streptococcus pyogenes after intramuscular injection in a diabetic patient: A case report
}

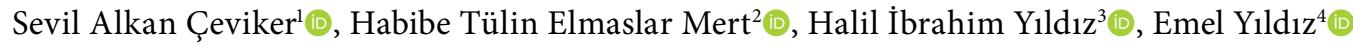 \\ ${ }^{1}$ Department of Infectious Diseases and Clinical Microbiology, University of Health Sciences Kütahya Evliya Çelebi Training and Research Hospital, Kütahya, Turkey \\ ${ }^{2}$ Department of Infectious Diseases and Clinical Microbiology, Trakya University Faculty of Medicine, Edirne, Turkey \\ ${ }^{3}$ Department of Anesthesiology and Reanimation, University of Health Sciences Kütahya Evliya Çelebi Training and Research Hospital, Kütahya, Turkey \\ ${ }^{4}$ Department of Orthopedics and Traumatology, University of Health Sciences Kütahya Evliya Çelebi Training and Research Hospital, Kütahya, Turkey
}

\begin{abstract}
Necrotizing fasciitis (NF) is the progressive necrosis of the skin and fascia and has morbidity and mortality rates of $70-80 \%$. The main causes of mortality are septic shock, multiorgan failure, and delay in diagnosis and treatment. Microorganisms responsible for NF are often group A Streptococci, Clostridium, and Bacteroides fragilis. Necrotizing fasciitis is a rare, life threatening soft tissue infection that requires emergency surgery and immediate medical attention. In this case report, we present a case of NF and necrotizing pneumonia caused by Streptococcus pyogenes who was admitted to our hospital with severe leg pain after intramuscular injection into the left gluteal region three days earlier.
\end{abstract}

Keywords: Necrotizing fasciitis, necrotizing pneumonia, Streptococcus pyogenes.

Necrotizing fasciitis (NF) is a rare, lifethreatening soft tissue infection that requires emergency surgery and immediate medical attention. ${ }^{[1,2]}$ Necrotizing fasciitis is also known as hemolytic streptococcus gangrene, Meleney ulcer, acute dermal gangrene, hospital gangrene, suppurative fasciitis, or synergistic necrotizing cellulitis. Necrotizing fasciitis may result from major traumatic injuries, minor damage to the skin or mucosa (e.g. abrasions, tears, or insect bites), varicella infection, non-penetrating soft tissue injuries, and obstetric and gynecological procedures. $^{[3]}$ In addition, it may occur as a complication of various surgical procedures or medical conditions such as cardiac catheterization, vascular sclerotherapy, diagnostic laparoscopy, or may be idiopathic. ${ }^{[4-8]}$ Necrotizing fasciitis is generally classified into three types according to the infectious agent.

a. Type I NF: polymicrobial with both aerobic and anaerobic bacteria; accounts for $80 \%$ of all NF cases

b. Type II NF: monomicrobial and usually caused by group A Streptococcus (GAS) (Streptococcus pyogenes [S. pyogenes])

c. Type III NF: caused by Vibrio or Aeromonas spp.; occurs with fulminant sepsis and rapid progression in patients exposed to contaminated water or food ${ }^{[3-7]}$

Despite advancements in surgical techniques and intensive care, NF still has high mortality rates. The main causes of mortality include septic

Received: November 18, 2019 Accepted: November 26, 2019 Published online: April 15, 2020

Correspondence: Sevil Alkan Çeviker, MD. SBÜ Kütahya Evliya Çelebi Eğitim ve Araştırma Hastanesi, Enfeksiyon Hastalıkları ve Klinik Mikrobiyoloji Kliniği, 43040 Kütahya, Türkiye. Tel: +90 506 - 6873768 e-mail: s-ewil@hotmail.com 
shock, multiorgan failure, and delayed diagnosis and treatment. ${ }^{[3,4,7,8]}$

In this case report, we present a case of NF and necrotizing pneumonia caused by $S$. pyogenes who was admitted to our hospital with severe leg pain after intramuscular (IM) injection into the left gluteal region three days earlier.

\section{CASE REPORT}

A 42-year-old female patient with no history of chronic disease other than diabetes mellitus (DM) presented to our outpatient clinic with complaints of swelling and redness in her left leg, pain, and high fever. The patient had received intramuscular injection into her left gluteal region due to lower back pain at the emergency department three days earlier. The patient stated she had pain in her leg for the past two days and did not present to the hospital. The patient's physical examination revealed she was in good general condition and conscious, body temperature: $38.4^{\circ} \mathrm{C}$, pulse: $103 \mathrm{bpm}$, Blood pressure (BP): 100/55 mmHg. Her left thigh and gluteal region showed hyperemia. Subcutaneous gas or crepitation was not present. Physical examination showed no additional pathologies. Laboratory tests revealed: White blood cells (WBC): $18,700 / \mathrm{mm}^{3}$, Hemoglobin (Hb): $11.2 \mathrm{~g} / \mathrm{dL}, \mathrm{C}$-reactive protein (CRP): $154 \mathrm{mg} / \mathrm{L}$, Creatine kinase (CK): $48 \mathrm{U} / \mathrm{L}$. Other tests were unremarkable. The patient was hospitalized with pre-diagnosis of cellulitis. X-ray and soft tissue ultrasound were performed, revealing no abscess or gas. Blood culture was taken before antibiotic

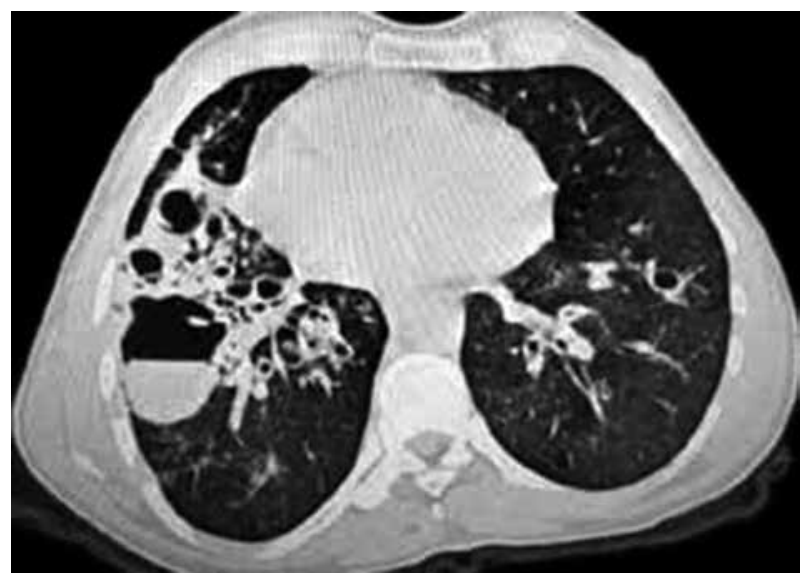

Figure 1. Bilateral necrotizing pneumonia. therapy. The patient was initiated empiric therapy of intravenous (IV) ampicillin-sulbactam $4 \times 1.5 \mathrm{gr} /$ day. On day three of treatment, the patients WBC, CRP, and CK levels elevated and the orthopedics was consulted. Lower limb computed tomography (CT) was performed and revealed subcutaneous emphysema of the left leg. With pre-diagnosis of NF, the patient was switched to empiric treatment of IV imipenem $4 \times 500 \mathrm{mg}+\mathrm{IV}$ clindamycin $3 \times 900 \mathrm{mg}$. The patient's clinical symptoms were unstable and the patient was transferred to the ICU. Thorax CT was performed for acute cough and difficulty breathing. The pulmonology specialist suspected necrotizing pneumonia (Figure 1). Deep tracheal aspirate (TA) culture was collected. Emergency lower left limb fasciotomy incision and soft tissue debridement were performed by the orthopedic specialist (Figure 2). Preoperative culture revealed $S$. pyogenes growth. Tracheal aspirate and blood culture also revealed $S$. pyogenes. Streptococcus pyogenes identification was evaluated at our hospital's microbiology laboratory with the VITEK 2 (BioMerieux, France) antibiogram system. Antibiogram results showed susceptibility to the patient's current treatment and the treatment was continued. In the postoperative period, the patient received daily wound dressing and wound debridement every two days. Follow debridement, the patient's clinical and laboratory findings and respiratory symptoms resolved and the patient was transferred to our hospital's Infectious Diseases ward. The patient received IV treatment for four weeks. Skin graft was performed and the patient was discharged in good health after a 42-day hospitalization period. Outpatient

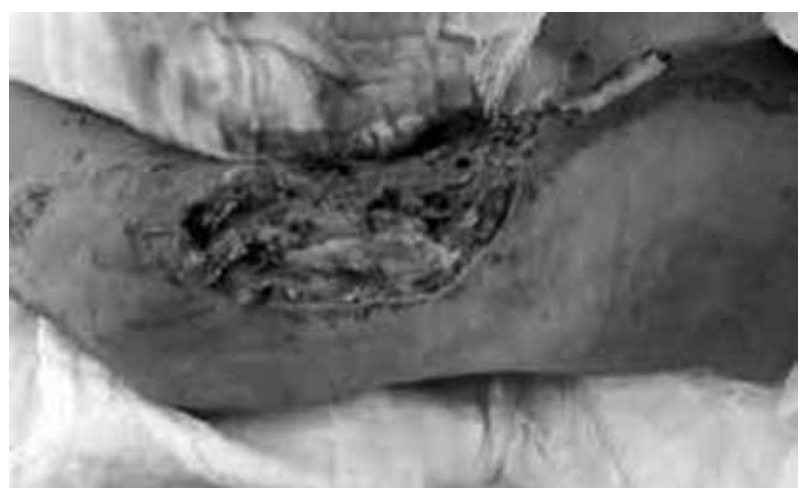

Figure 2. Appearance of left leg after debridement. 
follow-ups were uneventful. Informed consent was obtained from the patient's family.

\section{DISCUSSION}

According to the Lancefield grouping, $S$. pyogenes, which belongs to GAS, is one of the most important human pathogens of the streptococcus family. Invasive diseases that it causes in humans include bacteremia, pneumonia secondary to bacteremia, sepsis, $\mathrm{NF}$, streptococcal toxic shock syndrome (STSS), arthritis, and skin and soft tissue infections (SSTI). According to 2005 data, over 18 million people worldwide were estimated to be diagnosed with invasive $S$. pyogenes infection. ${ }^{[3-5]}$ Streptococcus pyogenes infections result in approximately 500,000 deaths per year, and significant morbidity and mortality rates worldwide. ${ }^{[9]}$ In developed countries, annual incidence of invasive infections is reported to be about 3-5/100,000. Mortality rate is reportedly $29 \%$, and even higher in those with STSS or septic shock (38\% and 45\%, respectively). ${ }^{[3,9]}$ The presented case is a case of pneumonia secondary to NF and bacteremia caused by $S$. pyogenes.

It has been reported that necrotizing fasciitis is most commonly seen in the abdominal wall, peritoneum and lower extremities and is more likely to occur in patients with DM, cancer, vascular disorder, immune deficiency, and chronic disease. ${ }^{[3,4,7,8]}$ In the present case, the patient had no known trauma or surgical history other than IM injection three days prior, though the patient had DM.

During the course of invasive diseases (especially NF) caused by $S$. pyogenes, immunosuppression is reported to develop and these patients become more vulnerable to secondary infections. ${ }^{[10]}$ Nawjin et al. ${ }^{[10]}$ reported that GAS necrotizing fasciitis patients were more likely to develop pneumonia during hospitalization compared to patients with NF of other causes. Pneumonia is especially likely to develop in the early term, regardless of young age and comorbid diseases. The case presented was a case of NF caused by $S$. pyogenes and pneumonia. Pneumonia was not present at admission but developed three days later, despite antibiotic treatment. It resolved after debridement and antibiotic therapy.
Early diagnosis and intervention of invasive GAS infection is of critical importance in reducing morbidity and mortality rates. When NF is present in patients with invasive GAS infection, severe pain is typical and occurs before shock or organ dysfunction. However, diagnosis and appropriate treatment may be delayed in patients taking analgesics or with diabetic neuropathy. ${ }^{[3]}$ In the case presented, leg pain was present at admission and exacerbation occurred despite treatment, as well as septic shock and necrotizing pneumonia.

Magnetic resonance imaging (MRI) and CT have been reported to be the most sensitive imaging methods for detecting air collections in soft tissues in NF cases. ${ }^{[1]}$ Although air was not detected in initial ultrasound or X-ray at admission, lower extremity CT on day three showed subcutaneous gas in the left leg, allowing NF diagnosis.

Management of invasive GAS infection is based on prompt diagnosis, appropriate antibiotic therapy, and, in some cases (e.g. NF), emergency surgical intervention. Mortality rate as high as $80 \%$ is reported in patients who do not undergo surgical intervention. ${ }^{[9,12]}$ In this case, the patient underwent debridement every other day.

In conclusion, NF is a clinical condition with rapid progression and high mortality rate and requires multidisciplinary evaluation. In the presented patient, $S$. pyogenes growth was observed in both deep TA and tissue cultures and the patient was treated with effective antibiotics and supportive therapies, and immediate and effective surgical intervention without developing mortality.

\section{Declaration of conflicting interests}

The authors declared no conflicts of interest with respect to the authorship and/or publication of this article.

\section{Funding}

The authors received no financial support for the research and/or authorship of this article.

\section{REFERENCES}

1. Green RJ, Dafoe DC, Raffin TA. Necrotizing fasciitis. Chest 1996;110:219-29.

2. Wong $\mathrm{CH}$, Chang HC, Pasupathy S, Khin LW, Tan JL, Low CO. Necrotizing fasciitis: clinical presentation, 
microbiology, and determinants of mortality. J Bone Joint Surg [Am] 2003;85:1454-60.

3. Stevens DL, Bryant AE. Necrotizing soft-tissue infections. N Engl J Med 2017;377:2253-65.

4. Hakkarainen TW, Kopari NM, Pham TN, Evans HL. Necrotizing soft tissue infections: review and current concepts in treatment, systems of care, and outcomes. Curr Probl Surg 2014;51:344-62.

5. Federman DG, Kravetz JD, Kirsner RS. Necrotizing fasciitis and cardiac catheterization. Cutis 2004;73:49-52.

6. Chan HT, Low J, Wilson L, Harris OC, Cheng AC, Athan E. Case cluster of necrotizing fasciitis and cellulitis associated with vein sclerotherapy. Emerg Infect Dis 2008;14:180-1.

7. Akcay EK, Cagil N, Yulek F, Anayol MA, Cetin H, Cag Y, et al. Necrotizing fasciitis of eyelid secondary to parotitis. Eur J Ophthalmol 2008;18:128-30.

8. Bharathan R, Hanson M. Diagnostic laparoscopy complicated by group A streptococcal necrotizing fasciitis. J Minim Invasive Gynecol 2010;17:121-3.

9. Nelson GE, Pondo T, Toews KA, Farley MM, Lindegren ML, Lynfield $\mathrm{R}$, et al. Epidemiology of Invasive Group A Streptococcal Infections in the United States, 2005-2012. Clin Infect Dis 2016;63:478-86

10. Nawijn F, Wassenaar ECE, Smeeing DPJ, Vlaminckx BJM, Reinders JSK, Wille J, et al. Exhaustion of the immune system by Group A Streptococcus necrotizing fasciitis: the occurrence of late secondary infections in a retrospective study. Trauma Surg Acute Care Open 2019;4:e000272.

11. Jiao L, Chagla Z, Kaki RM, Gohla G, Smieja M. Case report of necrotizing fasciitis associated with Streptococcus pneumoniae. Can J Infect Dis Med Microbiol 2016;2016:6872739.

12. Yamashiro E, Asato Y, Taira K, Awazawa R, Yamamoto Y, Hagiwara K, et al. Necrotizing fasciitis caused by Streptococcus pneumoniae. J Dermatol 2009;36:298-305. 